\title{
Towards integrated perioperative medicine: a survey of general practitioners' attitudes, beliefs and behaviours regarding perioperative medicine for older people
}

\author{
Authors: Tessa O'Halloran, ${ }^{\mathrm{A}}$ Jessie Colquhoun, ${ }^{\mathrm{B}}$ Gerard Danjoux, ${ }^{\mathrm{C}}$ Judith SL Partridge ${ }^{\mathrm{D}}$ and Jugdeep K Dhesi ${ }^{\mathrm{E}}$
}

\section{Background}

Perioperative optimisation can improve outcomes for older people having surgery. Integration with primary care could improve quality and reduce variability in access to preoperative optimisation.

Aim

Our aim was to explore attitudes, beliefs and behaviours of general practitioners (GPs) regarding the perioperative pathway, and evaluate enablers and barriers to GP-led preoperative optimisation.

Methods

Stakeholder interviews $(n=38)$ informed survey development. A purposive sampling frame was used to target delivery of online and paper surveys. Results were analysed using descriptive statistics.

Results

We had 231 responses (response rate 32.7\%). Enablers included belief among GPs that optimisation improves postoperative outcomes (86\%) and that they have a role discussing modifiable risk factors with patients (85\%). Barriers included low frequency exposure to older surgical patients, minimal training in perioperative medicine and rare interaction with perioperative services.

\section{Conclusion}

This survey illustrates the importance of interprofessional education, cross-sector training opportunities and collaboration to deliver integrated preoperative optimisation for older people undergoing surgery.

Authors: A geriatric medicine registrar, Guy's and St Thomas' NHS Foundation Trust, London, UK; ${ }^{\text {B }}$ general practice registrar Lewisham and Greenwich NHS Trust, London, UK; ${ }^{C}$ consultant in anaesthesia and sleep medicine, South Tees Hospitals NHS Foundation Trust, Middlesbrough, UK and honorary professor, Hull York Medical School, Hull, UK and Teeside University, Middlesbrough, UK; ' Consultant geriatrician, Guy's and St Thomas' NHS Foundation Trust, London, UK and honorary senior lecturer, King's College London, London, UK; Ehonorary reader, King's College London, London, UK and honorary associate professor, University College London, London, UK
KEYWORDS: perioperative medicine, primary care, integrated care, multimorbidity, transdisciplinary teams

DOI: $10.7861 /$ clinmed.2020-0851

\section{Introduction}

Increasing numbers of older people are having surgery. ${ }^{1}$ The advent of perioperative medicine has highlighted the need for closer collaboration between primary and secondary care, particularly in the management of older surgical patients who often have chronic health problems. While older people have much to gain from surgery, they are at high risk of adverse patient-reported, clinicianreported and process-related outcomes. ${ }^{2-4}$ Multidisciplinary preoperative optimisation services are increasingly advocated to mitigate this risk, with a growing evidence base to support improved postoperative outcomes and increasing attention to the expanding role of geriatricians in the care of the older surgical patient. ${ }^{5-8}$ Current models for preoperative optimisation are based within secondary care. From a clinical perspective, this model does not fully harness the expertise of primary care clinicians in optimising chronic health disease and promoting patient-led behaviour change; central tenets of preoperative optimisation. From a patient perspective, the transition of care between primary and secondary care has been highlighted for improvement in studies of experience-based codesign., ${ }^{9,10}$

The development of multidisciplinary preoperative optimisation services is supported by national reports and guidance. ${ }^{11-13}$ The initial evaluation of such secondary care services has resulted in a research recommendation from the National Institute for Health and Care Excellence. ${ }^{14}$ However, while these models are often based within secondary care, the NHS Long Term Plan advocates a proactive, prevention-focused health service spanning community and secondary care. ${ }^{15}$ Important advances toward more integrated surgical services include the Royal College of Anaesthetists' toolkit, Fitter Better Sooner, endorsed by the Royal College of General Practitioners (RCGP) as well as the Centre for Perioperative Care, in which the RCGP is a partner. ${ }^{16,17}$ The toolkit consists of patient resources and provides general practitioners (GPs) with an opportunity to use their expertise in empowering self-care, thus facilitating earlier preoperative optimisation in the surgical pathway. ${ }^{18}$ 
Potential benefits of early primary care-based optimisation for older people undergoing surgery include full utilisation of the preoperative optimisation window; patient-centred, place-based care; and continuity of care in chronic disease management, acknowledging the surgical episode as a punctuation in the longerterm patient journey. Additionally, faced with the forecasted surge in elective surgery following the COVID-19 pandemic, there is renewed urgency and opportunity for developing integrated models of place-based or virtual care that reduce reliance on hospital-based outpatient services. ${ }^{19}$

Despite these potential benefits, the perspective of primary care clinicians regarding perioperative medicine and their role in the surgical pathway has not been evaluated. This survey aimed to explore the attitudes, beliefs and behaviours of GPs pertaining to perioperative medicine. Specifically, the role of GPs in the surgical pathway, and interactions between primary and secondary care were examined. Additionally, the enablers and barriers to primary care-based optimisation of older surgical patients were described.

\section{Methods}

Ethics approval was not required for this study, as discussed with the lead trust research and development team.

A survey (supplementary material S1) was developed based on themes identified through 38 stakeholder interviews with healthcare professionals from primary and secondary care, ensuring representation from relevant specialties and disciplines. See supplementary material S2 for details of professional groups included.

The survey included multiple-choice, ranking, Likert, dichotomous and open-ended questions. It was reviewed for readability, non-ambiguity and validated by 12 expert raters from primary and secondary care. Content validity, calculated using Lawshe's method, was 0.79 which is above the validated threshold of 0.62 for 10 expert raters. ${ }^{20}$ The validated survey was piloted by a convenience sample of five GPs.

Data from NHS Digital were used to create a distribution curve of practice size, allowing definition of small, medium and large practices according to number of GP partners. Small practices were single-handed, medium practices had two to five GP partners, large practices had six to $10 \mathrm{GP}$ partners and mega practices employed more than $10 \mathrm{GP}$ partners. A purposive sampling frame ensured that practices of different sizes were represented in each of five geographical regions: the north; the midlands and the east; London; the south east; and the south west. Practices were then selected at random within each group. This approach to sampling aimed to deliver generalisable results in terms of practice size and location.

A bimodal delivery strategy was used, employing both online surveys and paper surveys between 15 January 2019 and 31 July 2019. Only fully trained GPs were targeted. A statement of participant information was provided via email invitation for online surveys, and on the covering page of paper surveys, with implied consent assumed on survey completion. SurveyMonkey was used to distribute online surveys through email invitation via practice managers, practice partners and direct to individual GPs, according to the preference of the GP practice. Three reminders were sent via email. To maximise response rates, paper surveys were also distributed at primary care meetings and educational events, where information regarding the size of the practice was available, in order to maintain fidelity to the sampling frame.
Survey results were analysed using basic descriptive statistics and reported by themes. It was beyond the scope of this work to formally analyse free text respondent views using qualitative methodology, but illustrative quotes are provided. See supplementary material S3 for all full text responses.

\section{Results}

\section{Response rate}

The survey was distributed to $706 \mathrm{GPs}$, with responses received from 231 (response rate of $32.7 \%$ ). Online surveys accounted for 187 responses (81\%), with a total of 44 paper survey responses (19\%). Clinicians from the London region comprised $53 \%$ of total respondents, and response rate varied by region (Fig 1). Most of the respondents were practice partners (59\%) followed by salaried GPs $(28 \%)$. Locum GPs made up $8 \%$ of respondents and the remaining $5 \%$ of respondents included GP registrars, one retainer $\mathrm{GP}$ and one nurse practitioner.

\section{Referral frequency to surgical specialties from primary care}

Over half of GP respondents had referred either zero (26\%) or one $(27 \%)$ patient over 65 years old to a surgical specialty in the past month. Forty per cent of respondents had referred two to five patients and $7 \%$ had referred six or more patients in the last month. Twenty per cent of GPs who had referred at least one patient to a surgical specialty in the past month reported that they perceived the risks of surgery to outweigh the benefits. Fiftyseven per cent reported having referred a patient for whom they could identify potentially modifiable risk factors for postoperative complications.

\section{Self-perceived role of primary care in the perioperative pathway}

Thirty-seven per cent of respondents reported that they had an important role in the surgical pathway. Eighty-seven per cent reported their role as a gatekeeper for referrals, with over $70 \%$ reporting a role in advocating for patients whom they felt would benefit from surgery (73\%) or from a non-operative alternative $(71 \%)$.

Eighty-five per cent of respondents believed it was their role to discuss modifiable risk factors for adverse perioperative outcome with patients. When faced with a hypothetical, high-risk, older

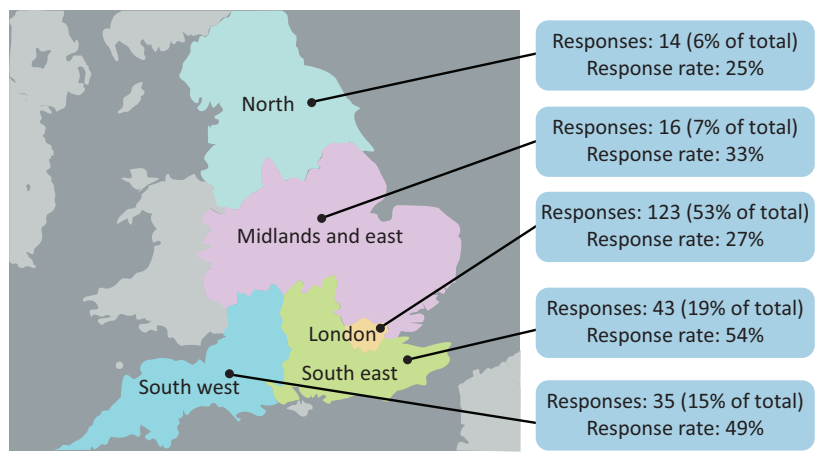

Fig 1. Survey response rate by region of England. 


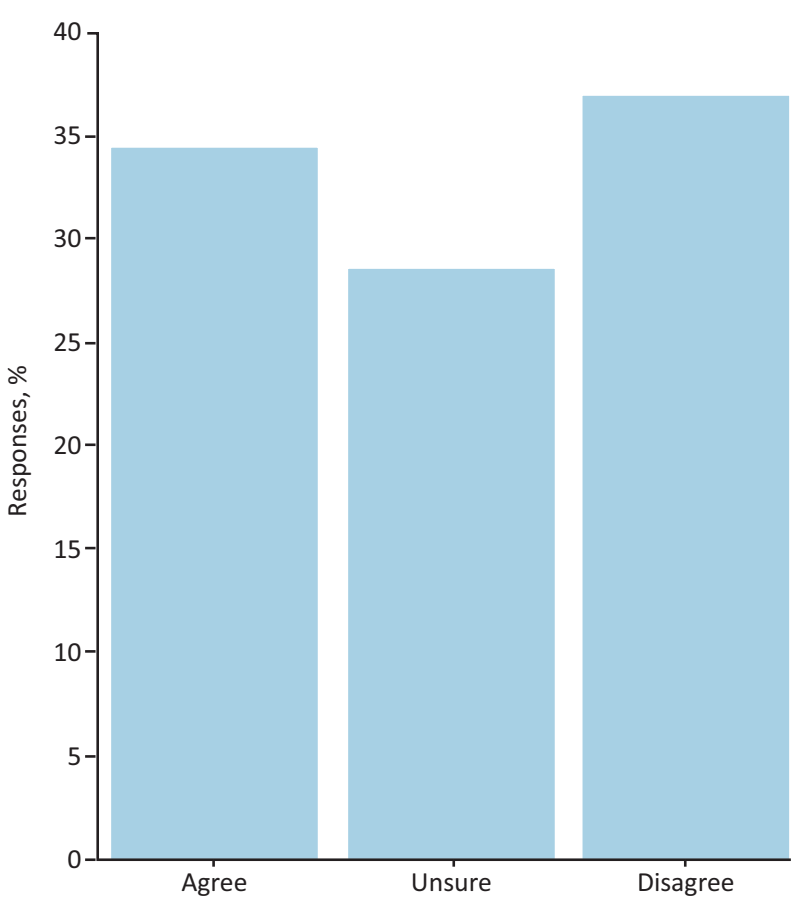

Fig 2. General practitioner responses to the statement: 'It is the role of secondary care to optimise patients for surgery.'

surgical patient, $58 \%$ of GPs reported that they would assess for modifiable risk factors that could be optimised before referring to a surgical team. Forty-four per cent of respondents reported that they would develop an optimisation plan for the patient. Lack of time was reported as a barrier to medically optimising patients by $45 \%$ of respondents. Fig 2 illustrates the varied views of respondents regarding whether it is the role of secondary care or primary care to preoperatively optimise patients for surgery.

Respondents reported that it was the role of the GP to engage in elements of shared decision making, including discussing alternatives to surgery (66\%), what would happen if they did not have surgery $(67 \%)$ and the perceived benefits of surgery $(70 \%)$. Despite this, fewer GPs reported enacting these behaviours in practice, with $61 \%$ discussing alternatives to surgery, $47 \%$ discussing what would happen if the patient did not have surgery and $54 \%$ discussing the perceived benefits of surgery. Some respondents reported that a lack of knowledge about surgical alternatives was a driver for this discrepancy (Box 1). GPs also reported that the necessary information to lead these conversations was not communicated from surgical specialties, with $73 \%$ reporting that alternatives to surgery were not clear from the surgical correspondence, with $64 \%$ reporting that decision making was not clearly communicated. GPs were asked if they explained the risks, benefits and alternatives to surgery with respect to the last patient they referred to a surgical specialty; Box 1 provides illustrative free-text answers.

\section{Interaction with surgical specialties and perioperative} medicine

Twenty-four per cent of GPs perceived that their assessment and evaluation of the patient was valued by the secondary care team.
Box 1. Discussion of factors important to shared decision making for surgery in the primary care setting: free text responses from general practitioners

'Limited to the knowledge I had on the subject.'

'Up to the point of knowledge for these issues, which is limited!'

'I refer to a surgeon after I have had a discussion with the patient about risks/benefits. The surgeon/anaesthetist is best placed to decide about suitability of a procedure, however, there is scanty information relayed to us about why a particular decision was made. So, when a patient comes back to discuss this, we have little information to base our discussion on.'

'I would only refer a patient for surgery if, in my opinion, the outcome would be beneficial or surgery is inevitable, but my discussion with the patient would be very much around modifiable factors and risk reduction / lifestyle counselling, if relevant.'

'I think GPs may overestimate the risk of surgery.'

'If a person has done research online and wants a referral for surgery, it is much quicker to accept and go ahead than try to dissuade. If you do, often you will be accused of trying to save money or it may be perceived that you are dismissing their problems.'

'I try as much as possible to discuss risks and benefits with patients, but am not always aware of all of the risks associated with certain procedures and therefore this can be difficult to do at times.'

Respondents reported a lack of clarity in communication from surgical specialties. In particular, $88 \%$ reported that the plan for preoperative management was not clear, with $84 \%$ reporting an unclear overall plan.

The majority of respondents agreed that preoperative medical optimisation of older patients can improve postoperative outcomes (86\%). Sixty-two per cent were unaware of perioperative medical services, although only $15 \%$ were aware that they may have access to geriatrician-led perioperative medicine services. A regional difference was noted in response to awareness of perioperative medical services; $4 \%$ of GPs from outside of London reported awareness of geriatrician-led perioperative medical services, compared with $27 \%$ of GPs from the London area. Additionally, $22 \%$ of GPs reported that they did not understand the role of perioperative medicine services. Again, a regional difference was apparent; $26 \%$ of GPs from outside London compared with $14 \%$ from London were unclear regarding the scope of perioperative medicine services.

\section{GP training in perioperative medicine}

Respondents reported a lack of training in perioperative medicine as a major barrier to GP-led preoperative optimisation, with just $12 \%$ of GPs reporting adequate training to deliver preoperative optimisation. Training in perioperative medicine was infrequent at all career stages, with most training undertaken at foundation level and the least training undertaken as GP registrars (Fig 3). 


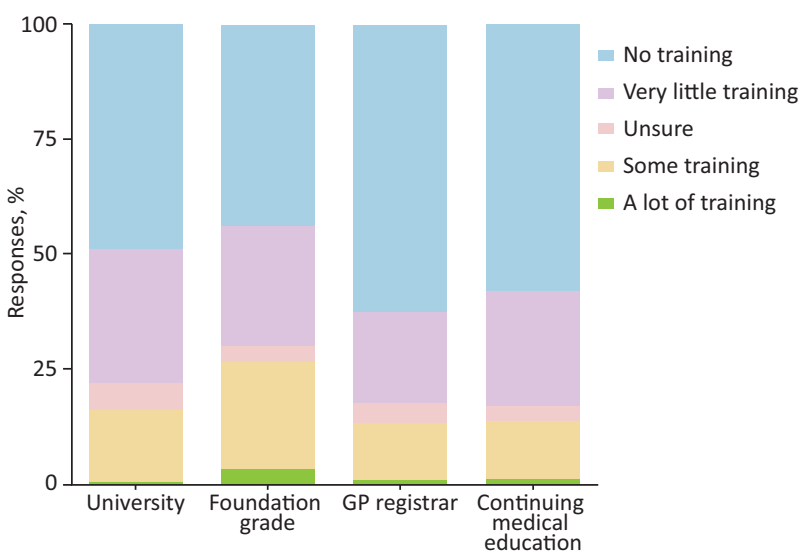

Fig 3. Perioperative medicine training at different stages of the general practitioner career span. GP = general practitioner.

\section{Enablers and barriers}

Key enablers and barriers to GP-led preoperative optimisation were described and grouped according to themes in Table 1.

\section{Discussion}

This is the first survey to explore the attitudes, beliefs and behaviours of GPs in England regarding perioperative medicine for older people, and to describe enablers and barriers to GP-based preoperative optimisation of older people undergoing surgery. The results showed that, although GPs overwhelmingly agree that preoperative optimisation of older patients can improve postoperative outcomes, there are significant cross-boundary clinical, process-related and professional barriers to implementing preoperative optimisation based in primary care. The COVID-19 pandemic has demonstrated how traditional barriers to innovative ways of working can be rapidly overcome. As health services adapt to the 'new normal', the opportunity to collaborate and integrate across the surgical pathway, from contemplation of surgery through to recovery, should be embraced by primary and secondary care providers.

It is widely acknowledged that the surgical population is ageing faster than the general population and surgical specialties are managing increasingly higher numbers of older, multimorbid patients. ${ }^{1,21}$ The role of primary care physicians in preoperative optimisation of longer-term conditions is frequently discussed given the contribution of such conditions to the perioperative risk profile in older patients undergoing surgery. ${ }^{22}$ Key potential advantages of GP-led optimisation include continuity in chronic disease management, GP expertise in empowering patient self-care and maximal use of lead time prior to surgery for timesensitive optimisation. ${ }^{22,23}$

However, the results of this survey illustrate the infrequency with which GPs refer older people to surgical specialties and the fact that this patient group represents a relatively small proportion of the vast primary care workload. Involvement of GPs in certain surgical pathways is likely reduced through direct surgical referrals from screening programmes (such as the national abdominal aortic aneurysm screening programme) and specialist community services (such as musculoskeletal pathways) that may bypass referral by GPs. Thus, managing the preoperative period can be an infrequent event for GPs and represents a small contributor to the daily workload in primary care. This may pose a limitation to quality improvement and process change, as both rely on regular exposure to a given event, to facilitate rapid feedback and change mechanisms. ${ }^{24}$

Perhaps, related to this low volume of exposure, training in perioperative medicine is relatively lacking throughout a career in general practice. Respondents reported feeling inadequately trained to provide preoperative medical optimisation for older patients, and believed a lack of knowledge was a barrier to engaging in shared decision making regarding the risks, benefits and alternatives to pursuing surgical treatment. Although perioperative medicine training programmes are emerging across the UK, these are targeting trainees from surgical, anaesthetic and general medicine backgrounds, with no targeted training opportunities for GP trainees. ${ }^{13,25-28}$ Furthermore, respondents reported minimal day-to-day interaction with perioperative medicine services and a lack of awareness of available services and the activities undertaken by such services. There is, however, geographical disparity in these findings, suggesting that there is an opportunity to reduce national variation. In addition, as virtual working becomes more commonplace following the COVID-19 pandemic, earlier involvement of GPs in perioperative optimisation may occur, thus increasing the opportunity for training and for collaborative care.

Finally, this survey revealed persistent professional barriers to GP-based preoperative optimisation. Respondents reported feeling that their assessment and evaluation of the patient was not valued

Table 1. Key enablers and barriers to general practitioner-led preoperative optimisation

Driver

'Buy-in'

Opportunity

Participation

Collaboration

GPs reported agreement that preoperative medical optimisation of older people can improve postoperative outcomes.

GPs reported identifying modifiable risk factors in many of the older patients referred to surgery

GPs reported that they have a role in discussing modifiable surgical risk factors with patients.

GPs reported that they have a role in shared decision making in the surgical pathway.

\section{Barriers}

Self-perception among GPs as having low importance in the surgical pathway.

Low frequency of exposure to older patients in the surgical pathway in routine practice.

Low levels of awareness of, and interaction with, perioperative medical services.

Low exposure to interdisciplinary training in perioperative medicine.

GPs $=$ general practitioners 
by the secondary care team. Fragmentation between primary and secondary care may have resulted in professional silos, with less robust relationships and less effective communication between healthcare settings. As such, shared interprofessional learning and relationship-building may be a key step in delivering integrated healthcare, mindful of a multimorbid population where acute admissions punctuate a patient journey shaped by long-term conditions. A good example of this, the Centre for Perioperative Care (CPOC) is a newly established cross-specialty organisation, with representation from all relevant colleges. CPOC aims to promote, advance and develop perioperative care through collaborative working. ${ }^{17}$ Such an approach with representation from the Royal College of General Practitioners is critical to address the challenges and embrace the opportunities described through this survey.

\section{Study limitations}

This study provides new knowledge regarding the role for GPs in perioperative care. A better understanding of the attitudes, beliefs and behaviours of GPs, and the barriers and enablers to GP-based preoperative optimisation can be used to develop collaborative integrated perioperative pathways. GPs were surveyed across all regions of England which allows a country-wide perspective of the challenges faced by GPs. However, this study is inherently limited as any survey of a large and heterogeneous group of clinicians, and should be interpreted as a first step to guiding further qualitative research and quality improvement initiatives. GP response rates to surveys are typically lower than those from the general medical professional population, with the overall response rate to this survey being consistent with other published surveys in primary care. ${ }^{29,30}$ The relatively small number of respondents (231) and variable regional response rate limits examination of differences between GPs' experience across regions. Of the five regions of England, 53\% of responses were from clinicians in London, thus other regions were less well represented. There is a possibility of response bias with respondents to the survey more likely to have an interest in, or experience of perioperative medicine services. Ambiguity of respondent interpretation of survey questions must be considered, although this was mitigated through piloting among GPs and validation with a group of expert raters.

Additionally, while this work focused on interprofessional factors, future work would benefit from patient and public representation to ensure that surgical pathways are patient-centred.

\section{Conclusion}

GP-based preoperative optimisation of older people could benefit patients and the health service, in line with the principles of the NHS Long Term Plan. Encouragingly, this survey has shown that GPs agree that preoperative optimisation can improve postoperative outcomes for older people, and that they have a role in identifying and managing modifiable risk factors. Future work should focus on interprofessional education, cross-sector training opportunities, relationship-building and clear role delineation in order to deliver quality integrated perioperative care for older people having surgery.

\section{Supplementary material}

Additional supplementary material may be found in the online version of this article at www.rcpjournals.org/clinmedicine:
S1 - Perioperative care of older people having surgery survey.

S2 - Professions of stakeholders interviewed in the development of the GP survey.

S3 - All free-text responses to questions with the option for a freetext entry.

\section{References}

1 Fowler AJ, Abbott TEF. Age of patients undergoing surgery. BJS 2019;106:1012-8.

2 Barnett K, Mercer SW, Norbury M. Epidemiology of multimorbidity and implications for health care, research, and medical education: a cross-sectional study. Lancet 2012;380:37-43.

3 Dasgupta M, Rolfson DB, Stolee P, Borrie MJ, Speechley M. Frailty is associated with postoperative complications in older adults with medical problems. Arch Gerontol Geriatr 2009;48:78-83.

4 Khuri S, Henderson W, DePalma R et al. Determinants of long-term survival after major surgery and the adverse effect of postoperative complications. Ann Surg 2005;242:326-43.

5 Partridge JS, Harari D, Martin FC et al. Randomised clinical trial of comprehensive geriatric assessment and optimization in vascular surgery. BJS 2017;104:679-87.

6 Joughin A, Partridge P, O'Halloran T, Dhesi J. Where are we now in perioperative medicine? Results from a repeated UK survey of geriatric medicine delivered services for older people. Age Ageing 2019:48:458-62.

7 Howie S, Tinker A. Are we on the same page? Exploring the role of the geriatrician in the care of the older surgical patient from the perspective of surgeons and geriatricians. Clin Med 2018;18:374-9.

8 Harari D, Hopper A, Dhesi ] et al. Proactive care of older people undergoing surgery ('POPS'): designing, embedding, evaluating and funding a comprehensive geriatric assessment service for older elective surgical patients. Age Ageing 2007;36:190-6.

9 Grocott M. Pathway redesign: putting patients ahead of professionals. Clin Med 2019;19:468-72.

10 Tsianakas V, Robert R, Maben J, Richardson A et al. Implementing patient-centred cancer care: using experience-based co-design to improve patient experience in breast and lung cancer services. Support Care Cancer 2012;20:2649.

11 Wilkinson K, Martin IC, Gough M] et al. An age old problem: A review of the care received by elderly patients undergoing surgery. National Confidential Enquiry into Patient Outcome and Death, 2010.

12 Findlay G, Goodwin A, Protopapa K, Smith N, Mason M. Knowing the risk: A review of the peri-operative care of surgical patients. National Confidential Enquiry into Patient Outcome and Death, 2011.

13 Griffiths R, Beech F, Brown A et al. Peri-operative care of the elderly 2014: Association of Anaesthetists of Great Britain and Ireland. Anaesthesia 2014;69(Suppl 1):81-98.

14 National Institute for Health and Care Excellence. Perioperative care in adults: NICE guideline [NG180]. NICE, 2020. www.nice.org. uk/guidance/NG180 [Accessed 12 December 2020].

15 NHS England. The NHS Long Term Plan. NHS, 2019. www. longtermplan.nhs.uk [Accessed 13 July 2019].

16 Royal College of Anaesthetists. Fitter Better Sooner. RCoA, 2018. www.rcoa.ac.uk/patient-information/preparing-surgery-fitter-bettersooner [Accessed 15 January 2020].

17 Centre for Perioperative Care. About CPOC. CPOC. https://cpoc.org. uk/about-cpoc [Accessed 04 February 2020].

18 Royal College of General Practitioners. The RCGP curriculum. RCGP, 2019. www.rcgp.org.uk/-/media/Files/GP-training-and-exams/ Curriculum-2019/Curriculum-Topic-Guides-300819.ashx?la=en [Accessed 15 January 2020].

19 Spinelli A, Pellino G. COVID-19 Pandemic: perspectives on an unfolding crisis. BJS 2020;107:785-878. 
20 Lawshe $\mathrm{CH}$. A quantitative approach to content validity. Pers Psychol 1975;28:563.

21 Klopfenstein CE, Herrmann FR, Michel JP, Clergue F, Forster A. The influence of an aging surgical population on the anesthesia workload: a ten-year survey. Anesth Analg 1998;86:1165-70.

22 Royal College of Anaesthetisis. Perioperative medicine pathway to better surgical care. RCoA, 2015. www.rcoa.ac.uk/sites/default/files/ documents/2019-08/PERIOP-2014.pdf [Accessed 6 June 2020]

23 Deeney S, Thorlby R, Steventon A. Reducing emergency admissions. The Health Foundation, 2018.

24 Foot C, Naylor C, Imison C. The quality of GP diagnosis and referral. The King's Fund, 2019.

25 Intercollegiate Surgical Curriculum Programme. General surgery curriculum. ISCP, 2016. www.iscp.ac.uk/static/public/syllabus/syllabus_gs_2016.pdf [Accessed 11 December 2019].

26 Joint Royal Colleges of Physicians Training Board. Specialty training curriculum for geriatric medicine curriculum. JRCPTB, 2016.

27 Rogerson A, Partridge J, Dhesi J. A foundation programme educational placement in peri-operative medicine for older people: mixed methods evaluation. Anaesthesia 2018;73:1392-9.
28 Barber S, Singleton E, Partridge J, Dhesi J. Training doctors in perioperative medicine for older people undergoing surgery (POPS): an innovative foundation placement. Clin Med 2019;19:465-7.

29 Asch DA, Jedrziewski MK, Christakis NA. Response rates to mail surveys published in medical journals. J Clin Epidemiol 1997;50: 1129-36.

30 Bonevski B, Magin P, Horton G, Foster M, Girgis A. Response rates in GP surveys - trialling two recruitment strategies. Aust Fam Physician 2011:40:427-30.

Address for correspondence: Dr Tessa O'Halloran, Department of Ageing and Health, Guy's Hospital, Guy's and St Thomas' NHS Foundation Trust, Great Maze Pond, London SE1 9RT, UK. Email: tessa.ohalloran@gmail.com

Twitter: @JKDhesi 